\title{
AN OPTIMAL MATCHING PROBLEM
}

\author{
IVAR EKELAND ${ }^{1}$
}

\begin{abstract}
Given two measured spaces $(X, \mu)$ and $(Y, \nu)$, and a third space $Z$, given two functions $u(x, z)$ and $v(x, z)$, we study the problem of finding two maps $s: X \rightarrow Z$ and $t: Y \rightarrow Z$ such that the images $s(\mu)$ and $t(\nu)$ coincide, and the integral $\int_{X} u(x, s(x)) d \mu-\int_{Y} v(y, t(y)) d \nu$ is maximal. We give condition on $u$ and $v$ for which there is a unique solution.
\end{abstract}

Mathematics Subject Classification. 05C38, 15A15, 05A15, 15A18.

Received February 4, 2004.

\section{IntRoduction}

In the economic theory of hedonic pricing (see [5] for an overview), one encounters the following problem. We are given two sets $X$ and $Y$, endowed with positive measures $\mu$ and $\nu$ such that $\mu(X)=\nu(Y)$. We wish to transport them into a third set $Z$, so that each element $x \in X$ is matched with an element $y \in Y$, and the resulting pair is located at some point $z \in Z$. The cost of transporting $x$ to $z$ is $w(x, z)$ and the cost of transporting $y$ to $z$ is $v(y, z)$. Find the assignments $z=s(x)$ and $z=t(y)$ which will minimize the total cost of transportation.

This translates into the following optimization problem: find maps $s: X \rightarrow Z$ and $t: Y \rightarrow Z$ such that $s(\mu)=t(\nu)$ (this is the matching condition) and the integral

$$
\int_{X} w(x, s(x)) \mathrm{d} \mu+\int_{Y} v(y, t(y)) \mathrm{d} \nu
$$

is minimized.

The economics of this problem will be dealt with elsewhere. The purpose of this paper is to deal with the mathematical aspects, which are interesting in themselves. Clearly, it is related to the classical optimal transportation (or mass transfer) problem of Monge and Kantorovitch. Recall that this problem consists in minimizing the integral

$$
\int_{X} u(x, \varphi(x)) \mathrm{d} \mu
$$

among all maps $\varphi: X \rightarrow Y$ such that $\varphi(\mu)=\nu$. Here the measured spaces $(X, \mu)$ and $(Y, \nu)$ are given, with $\mu(X)=\nu(Y)$, as well as the function $u: X \times Y \rightarrow R$. We refer to the monographs [7] or [8] for recent accounts of the theory.A basic result by Brenier [1] states that, if $X$ and $Y$ are bounded open subsets of $R^{n}$ endowed with the Lebesgue measure, with $X$ connected, and $u(x, y)=\|x-y\|^{2}$, then there is a unique solution $\varphi$ to

Keywords and phrases. optimal transportation, measure-preserving maps.

1 University of British Columbia, Vancouver BC, V6T 1Z2 Canada. e-mail: ekeland@math.ubc.ca 
the optimal transportation problem, and $\varphi$ is almost everywhere equal to the gradient of a convex function. More generally, if $u$ is continuously differentiable up to and including the boundary of $X \times Y$, and if the partials $D_{x} u(x, \cdot)$ and $D_{y} u(\cdot, y)$ are one-to-one:

$$
\begin{aligned}
& D_{x} u\left(x, y_{1}\right)=D_{x} u\left(x, y_{2}\right) \Longrightarrow y_{1}=y_{2} \\
& D_{y} u\left(x_{1}, y\right)=D_{y} u\left(x_{2}, y\right) \Longrightarrow x_{1}=x_{2}
\end{aligned}
$$

then the optimal mass transportation problem has a solution.

A first approach to the optimal matching problem would be as follows. Define a function $u: X \times Y \rightarrow R$ by:

$$
u(x, y)=\min _{z}\{w(x, z)+v(\varphi(x), z)\}
$$

and seek a measure-preserving map $\varphi: X \rightarrow Y$ which minimizes the integral (1.2). We then recover the map $s: X \rightarrow Z$ by setting:

$$
w(x, s(x))+v(\varphi(x), s(x))=\min _{z}\{w(x, z)+v(\varphi(x), z)\}=u(x, \varphi(x))
$$

and since $\varphi$ is measure-preserving, it is invertible up to a subset of measure zero, so that we recover $t: Y \rightarrow Z$ by the formula $t(y)=s\left(\varphi^{-1}(y)\right)$.

This approach will succeed whenever the function $u$ defined by formula (1.5) is continuously differentiable and satisfies condition (1.3) and (1.4). Unfortunately, this is far from being the general case. Another method must be devised to solve the optimal matching problem, and it is the purpose of the present paper.

Kantorovitch [6] introduced into the optimal transportation problem a duality method which will be crucial to our proof. Instead of proving directly existence and uniqueness in the optimal matching problem, we solve in Section 3 another optimization problem, and we will show in Section 4 that it yields the solution to the original one. This correspondence relies heavily on an extension of the classical duality results in convex analysis (see [3]). This extension can be found in [2,4,7], or [8]; for the reader's convenience, we provide a short but complete treatment in Section 2. Finally, in the last sections, we will give some examples.

\section{The MAIN RESUlT}

From now on, $X \subset R^{n_{1}}, Y \subset R^{n_{2}}$, and $Z \subset R^{n_{3}}$ will be compact subsets. We are given measures $\mu$ on $X$ and $\nu$ on $Y$, which are absolutely continuous with respect to the Lebesgue measure, and satisfy:

$$
0<\mu(X)=\nu(Y)<\infty .
$$

We are also given functions $u: \Omega_{1} \rightarrow R$ and $v: \Omega_{2} \rightarrow R$, where $\Omega_{1}$ is a neighbourhood of $X \times Z$ and $\Omega_{2}$ is a neigbourhood of $Y \times Z$. It is assumed that $u$ and $v$ are continuous with respect to both variables, differentiable with respect to $x$ and $y$, and that the partial derivatives $D_{x} u$ and $D_{y} v$ are continuous with respect to both variables, and injective with respect to $z$ :

$$
\begin{aligned}
& \forall x \in X, D_{x} u\left(x, z_{1}\right)=D_{x} u\left(x, z_{2}\right) \Longrightarrow z_{1}=z_{2} \\
& \forall y \in Y, D_{y} v\left(y, z_{1}\right)=D_{y} v\left(y, z_{2}\right) \Longrightarrow z_{1}=z_{2} .
\end{aligned}
$$

The latter condition is a generalization of the classical Spence-Mirrlees condition in the economics of asymmetric information (see [2]). It is satisfied for $u(x, z)=\|x-z\|^{\alpha}$, provided $\alpha \neq 0$ and $\alpha \neq 1$. If $\alpha<1$ we will also require that $X \cap Y=\varnothing$ 
Theorem 1. Under the above assumptions, there exists a pair of Borelian maps $(\bar{s}, \bar{t})$ with $\bar{s}(\mu)=\bar{t}(\nu)$, such that for every $(s, t)$ satisfying $s(\mu)=t(\nu)$, we have:

$$
\int_{X} u(x, s(x)) \mathrm{d} \mu-\int_{Y} v(y, t(y)) \mathrm{d} \nu \leq \int_{X} u(x, \bar{s}(x)) \mathrm{d} \mu-\int_{Y} v(y, \bar{t}(y)) \mathrm{d} \nu<\infty .
$$

This solution is unique, up to equality almost everywhere, and it is described as follows: there is some Lipschitz continuous function $\bar{p}: Z \rightarrow R$ and some negligible subsets $X_{0} \subset X$ and $Y_{0} \subset Y$ such that, for every $x \notin X_{0}$ and every $y \notin Y_{0}$ :

$$
\begin{aligned}
& \forall z \neq \bar{s}(x), \quad u(x, z)-\bar{p}(z)<u(x, \bar{s}(x))-\bar{p}(\bar{s}(x)) \\
& \forall z \neq \bar{t}(y), \quad v(y, z)-\bar{p}(z)>v(x, \bar{t}(y))-\bar{p}(\bar{t}(y)) \\
& \bar{p} \text { is differentiable at } \bar{s}(x) \text { and } \bar{t}(y) .
\end{aligned}
$$

If in addition $u$ and $v$ are differentiable with respect to $z$, we get, from the minimization $(2.3)$ and the maximization (2.4):

$$
\begin{aligned}
D_{z} u(x, \bar{s}(x)) & =D_{z} \bar{p}(\bar{s}(x)) \\
D_{z} v(y, \bar{t}(y)) & =D_{z} \bar{p}(\bar{t}(y)) .
\end{aligned}
$$

Set $\bar{s}(\mu)=\bar{t}(\nu)=\lambda$. It follows from the above that, for $\lambda$-almost every $z \in Z$, there is some $x \notin X_{0}$ and $y \notin Y_{0}$ such that $z=\bar{s}(x)=\bar{t}(y)$, and for every such $(x, y)$ we have:

$$
D_{z} u(x, z)=D_{z} \bar{p}(z)=D_{z} v(y, z)
$$

Note that there is no reason why $\lambda$ should be absolutely continuous with respect to the Lebesgue measure.

The proof ot Theorem $1 \mathrm{~s}$ deferred to Section 4. Meanwhile, let us notice that we have slightly changed the formulation of the optimal matching problem: by setting $w=-u$ we recover the original one. This change will simplify future notations.

\section{FUnDAMENTALS OF $u$-CONVEX ANALYSIS}

In this section, we basically follow Carlier [2].

\section{1. $u$-convex functions}

We will be dealing with function taking values in $\mathbb{R} \cup\{+\infty\}$.

A function $f: X \rightarrow \mathbb{R} \cup\{+\infty\}$ will be called $u$-convex iff there exists a non-empty subset $A \subset Z \times \mathbb{R}$ such that:

$$
\forall x \in X, \quad f(x)=\sup _{(z, a) \in A}\{u(x, z)+a\} .
$$

A function $p: Z \rightarrow \mathbb{R} \cup\{+\infty\}$ will be called $u$-convex iff there exists a non-empty subset $B \subset X \times \mathbb{R}$ such that:

$$
p(z)=\sup _{(x, b) \in B}\{u(x, z)+b\}
$$

\subsection{Subconjugates}

Let $f: X \rightarrow \mathbb{R} \cup\{+\infty\}$, not identically $\{+\infty\}$, be given. We define its subconjugate $f^{\sharp}: Z \rightarrow \mathbb{R} \cup\{+\infty\}$ by:

$$
f^{\sharp}(z)=\sup _{x}\{u(x, z)-f(x)\} \cdot
$$


It follows from the definitions that $f^{\sharp}$ is a $u$-convex function on $Z$ (it might be identically $\{+\infty\}$ ).

Let $p: Z \rightarrow \mathbb{R} \cup\{+\infty\}$, not identically $\{+\infty\}$, be given. We define its subconjugate $p^{\sharp}: X \rightarrow \mathbb{R} \cup\{+\infty\}$ by:

$$
p^{\sharp}(x)=\sup _{z}\{u(x, z)-p(z)\} .
$$

It follows from the definitions that $p^{\sharp}$ is a $u$-convex function on $X$ (it might be identically $\{+\infty\}$ ).

Example 1. Set $f(x)=u(x, \bar{z})+a$. Then

$$
f^{\sharp}(\bar{z})=\sup _{x}\{u(x, \bar{z})-u(x, \bar{z})-a\}=-a .
$$

Conjugation reverses ordering: if $f_{1} \leq f_{2}$, then $f_{1}^{\sharp} \geq f_{2}^{\sharp}$, and if $p_{1} \leq p_{2}$, then $p_{1}^{\sharp} \geq p_{2}^{\sharp}$. As a consequence, if $f$ is $u$-convex, not identically $\{+\infty\}$, then $f^{\sharp}$ is $u$-convex, not identically $\{+\infty\}$, . Indeed, since $f$ is $u$-convex, we have $f(x) \geq u(x, z)+a$ for some $(z, a)$, and then $f^{\sharp}(z) \leq-a<\infty$.

Proposition 1 (the Fenchel inequality). For any functions $f: X \rightarrow \mathbb{R} \cup\{+\infty\}$ and $p: X \rightarrow \mathbb{R} \cup\{+\infty\}$, not identically $\{+\infty\}$, we have:

\subsection{Subgradients}

$$
\begin{aligned}
& \forall(x, z), f(x)+f^{\sharp}(z) \geq u(x, z) \\
& \forall(x, z) \quad p(z)+p^{\sharp}(x) \geq u(x, z) .
\end{aligned}
$$

Let $f: X \rightarrow \mathbb{R} \cup\{+\infty\}$ be given, not identically $\{+\infty\}$. Take some point $x \in X$. We shall say that a point $z \in Z$ is a subgradient of $f$ at $x$ if the points $x$ and $z$ achieve equality in the Fenchel inequality:

$$
f(x)+f^{\sharp}(z)=u(x, z) .
$$

The set of subgradients of $f$ at $x$ will be called the subdifferential of $f$ at $x$ and denoted by $\partial f(x)$.

Similarly, let $p: Z \rightarrow \mathbb{R} \cup\{+\infty\}$ be given, not identically $\{+\infty\}$. Take some point $z \in Z$. We shall say that a point $x \in X$ is a subgradient of $p$ at $z$ if:

$$
p^{\sharp}(x)+p(z)=u(x, z) .
$$

The set of subgradients of $p$ at $z$ will be called the subdifferential of $p$ at $z$ and denoted by $\partial p(z)$.

Proposition 2. The following are equivalent:

(1) $z \in \partial f(x)$;

(2) $\forall x^{\prime}, f\left(x^{\prime}\right) \geq f(x)+u\left(x^{\prime}, z\right)-u(x, z)$.

If equality holds for some $x^{\prime}$, then $z \in \partial f\left(x^{\prime}\right)$ as well.

Proof. We begin with proving that the first condition implies the second one. Assume $z \in \partial f(x)$. Then, by (3.5) and the Fenchel inequality, we have:

$$
f\left(x^{\prime}\right) \geq u\left(x^{\prime}, z\right)-f^{\sharp}(z)=u\left(x^{\prime}, z\right)-[u(x, z)-f(x)] .
$$

We then prove that the second condition implies the first one. Using the inequality, we have:

$$
\begin{aligned}
f^{\sharp}(z) & =\sup _{x^{\prime}}\left\{u\left(x^{\prime}, z\right)-f\left(x^{\prime}\right)\right\} \\
& \leq \sup _{x^{\prime}}\left\{u\left(x^{\prime}, z\right)-f(x)-u\left(x^{\prime}, z\right)+u(x, z)\right\} \\
& =u(x, z)-f(x)
\end{aligned}
$$

so $f(x)+f^{\sharp}(z) \leq u(x, z)$. We have the converse by the Fenchel inequality, so equality holds. 
Finally, if equality holds for some $x^{\prime}$ in condition (2), then $f\left(x^{\prime}\right)-u\left(x^{\prime}, z\right)=f(x)-u(x, z)$, so that:

$$
\begin{aligned}
\forall x^{\prime \prime}, \quad f\left(x^{\prime \prime}\right) & \geq f(x)-u(x, z)+u\left(x^{\prime \prime}, z\right) \\
& =f\left(x^{\prime}\right)-u\left(x^{\prime}, z\right)+u\left(x^{\prime \prime}, z\right)
\end{aligned}
$$

which implies that $z \in \partial f\left(x^{\prime}\right)$.

There is a similar result for functions $p: Z \rightarrow \mathbb{R} \cup\{+\infty\}$, not identically $\{+\infty\}$ : we have $x \in \partial p(z)$ if and only if

$$
\forall\left(x^{\prime}, z^{\prime}\right), \quad p\left(z^{\prime}\right) \geq p(z)+u\left(x, z^{\prime}\right)-u(x, z) .
$$

\subsection{Biconjugates}

It follows from the Fenchel inequality that, if $p: Z \rightarrow \mathbb{R} \cup\{+\infty\}$ is not identically $\{+\infty\}$ :

$$
p^{\sharp \sharp}(z)=\sup _{x}\left\{u(x, z)-p^{\sharp}(x)\right\} \leq p(z) .
$$

Example 2. Set $p(z)=u(\bar{x}, z)+b$. Then

$$
\begin{aligned}
p^{\sharp \sharp}(z) & =\sup _{x}\left\{u(x, z)-p^{\sharp}(x)\right\} \\
& \geq u(\bar{x}, z)-p^{\sharp}(\bar{x}) \\
& =u(\bar{x}, z)+b=p(z) .
\end{aligned}
$$

This example generalizes to all $u$-convex functions. Denote by $C_{u}(Z)$ the set of all $u$-convex functions on $Z$.

Proposition 3. For every function $p: Z \rightarrow \mathbb{R} \cup\{+\infty\}$, not identically $\{+\infty\}$, we have

$$
p^{\sharp \sharp}(z)=\sup _{\varphi}\left\{\varphi(z) \mid \varphi \leq p, \varphi \in \mathbb{C}_{u}(Z)\right\} .
$$

Proof. Denote by $\bar{p}(z)$ the right-hand side of the above formula. We want to show that $p^{\sharp \sharp}(z)=\bar{p}(z)$.

Since $p^{\sharp \sharp} \leq p$ and $p^{\sharp \sharp}$ is $u$-convex, we must have $p^{\sharp \sharp} \leq \bar{p}$.

On the other hand, $\bar{p}$ is $u$-convex because it is a supremum of $u$-convex functions. So there must be some $B \subset X \times \mathbb{R}$ such that:

$$
p(z)=\sup _{(x, b) \in B}\{u(x, z)+b\} .
$$

Let $(x, b) \in B$. Since $\bar{p} \leq p$, we have $u(x, z)+b \leq \bar{p}(z) \leq p(z)$. Taking biconjugates, as in the preceding example, we get $u(x, z)+b \leq p^{\sharp \sharp}(z)$. Taking the supremums over $(x, b) \in B$, we get the desired result.

Corollary 1. Let $p: Z \rightarrow \mathbb{R} \cup\{+\infty\}$ be a u-convex function, not identically $\{+\infty\}$. Then $p=p^{\sharp}$, and the following are equivalent:

(1) $x \in \partial p(z)$;

(2) $p(z)+p^{\sharp}(x)=u(x, z)$;

(3) $z \in \partial p^{\sharp}(x)$.

Proof. We have $p^{\sharp \sharp} \leq p$ always by relation (3.8). Since $p$ is $u$-convex, we have:

$$
p(z)=\sup _{(x, b) \in B}\{u(x, z)+b\}
$$


for some $B \subset X \times \mathbb{R}$. By Proposition 3, we have:

$$
\sup _{(x, b) \in B}\{u(x, z)+b\} \leq p^{\sharp \sharp}(z)
$$

and so we must have $p=p^{\sharp \sharp}$. Taking this relation into account, as well as the definition of the subgradient, we see that condition (2) is equivalent both to (1) and to (2)

Definition 1. We shall say that a function $p: Z \rightarrow \mathbb{R} \cup\{+\infty\}$ is $u$-adapted if it is not identically $\{+\infty\}$ and there is some $(x, b) \in X \times R$ such that:

$$
\forall z \in Z, \quad p(z) \geq u(x, z)+b
$$

It follows from the above that if $p$ is $u$-adapted, then so are $p^{\sharp}, p^{\sharp \sharp}$ and all further subconjugates. Note that a $u$-convex function which is not identically $\{+\infty\}$ is $u$-adapted.

Corollary 2. Let $p: Z \rightarrow \mathbb{R} \cup\{+\infty\}$ be u-adapted. Then:

$$
p^{\sharp \sharp \#}=p^{\sharp} .
$$

Proof. If $p$ is $u$-adapted, then $p^{\sharp}$ is $u$-convex and not identically $\{+\infty\}$. The result then follows from Corollary 1 .

\subsection{Smoothness}

Since $u$ is continuous and $X \times Z$ is compact, the family

$$
\{u(x, \cdot) \mid x \in X\}
$$

is uniformly equicontinuous on $Z$. It follows from definition 3.1 that all $u$-convex functions on $Z$ are continuous (in particular, they are finite everywhere).

Denote by $k$ the upper bound of $\left\|D_{x} u(x, z)\right\|$ for $(x, z) \in X \times Z$. Since $D_{x} u$ is continuous and $X \times Z$ is compact, we have $k<\infty$, and the functions $x \rightarrow u(x, z)$ are all $k$-Lipschitzian on $X$. Again, it follows from Definition 3.1 that all $u$-convex functions on $X$ are $k$-Lipschitz (in particular, they are finite everywhere). By Rademacher' theorem, they are differentiable almost everywhere with respect to the Lebesgue measure.

Let $f: X \rightarrow R$ be $u$-convex. Since $f=f^{\sharp \sharp}$, we have:

$$
f(x)=\sup _{z}\left\{u(x, z)-f^{\sharp}(z)\right\} .
$$

Since $f^{\sharp}$ is $u$-convex, it is continuous, and the supremum is achieved on the right-hand side, at some point $z \in \partial f(x)$. This means that all $u$-convex functions on $X$ are subdifferentiable everywhere on $X$.

Let $x$ be a point where $f$ is differentiable, with derivative $D_{x} f(x)$, and let $z \in \partial f(x)$. Consider the function $\varphi\left(x^{\prime}\right)=u\left(x^{\prime}, z\right)-f^{\sharp}(z)$. By Proposition 2, we have $\varphi \leq f$ and $\varphi(x)=f(x)$, so that $\varphi$ and $f$ must have the same derivative at $x$ :

$$
D_{x} f(x)=D_{x} u(x, z) .
$$

By condition (2.1), this equation defines $z$ uniquely. We shall denote it by $z=\nabla_{u} f(x)$. In other words, at every point $x$ where $f$ is differentiable, the subdifferential $\partial f(x)$ reduces to a singleton, namely $\left\{\nabla_{u} f(x)\right\}$. Combining all this information, we get:

Proposition 4. For every u-convex function $f: X \rightarrow R$, there is a map $\nabla_{u} f: X \rightarrow Z$ such that, for almost every $x$ :

$$
D_{x} f(x)=D_{x} u(x, z) \Longleftrightarrow z=\nabla_{u} f(x) .
$$


The following result will also be useful:

Proposition 5. Let $p: Z \rightarrow R$ be u-adapted, and let $x \in X$ be given. Then there is some point $z \in \partial p^{\sharp}(x)$ such that $p(z)=p^{\sharp \sharp}(z)$.

Proof. Assume otherwise, so that for every $z \in \partial p^{\sharp}(x)$ we have $p^{\sharp \sharp}(z)<p(z)$. For every $z \in \partial p^{\sharp}(x)$, we have $x \in \partial p^{\sharp \sharp}(z)$, so that, by Proposition 2, we have

$$
p^{\sharp \sharp}\left(z^{\prime}\right) \geq u\left(x, z^{\prime}\right)-u(x, z)+p^{\sharp \sharp}(z)
$$

for all $z^{\prime} \in Z$, the inequality being strict if $z^{\prime} \notin \partial p^{\sharp}(x)$. Set $\varphi_{z}\left(z^{\prime}\right)=u\left(x, z^{\prime}\right)-u(x, z)+p^{\sharp \sharp}(z)$. We have:

$$
\begin{aligned}
& z^{\prime} \notin \partial p^{\sharp}(x) \Longrightarrow \varphi_{z}\left(z^{\prime}\right)<p^{\sharp \sharp}\left(z^{\prime}\right) \leq p\left(z^{\prime}\right) \\
& z^{\prime} \in \partial p^{\sharp}(x) \Longrightarrow \varphi_{z}\left(z^{\prime}\right) \leq p^{\sharp \sharp}\left(z^{\prime}\right)<p\left(z^{\prime}\right)
\end{aligned}
$$

so that $\varphi_{z}\left(z^{\prime}\right)<p\left(z^{\prime}\right)$ for all $\left(z, z^{\prime}\right)$. Since $Z$ is compact, there is some $\varepsilon>0$ such that $\varphi_{z}\left(z^{\prime}\right)+\varepsilon \leq p\left(z^{\prime}\right)$ for all $\left(z, z^{\prime}\right)$. Taking the subconjugate with respect to $z^{\prime}$, we get:

$$
\begin{aligned}
p^{\sharp}(x) & \leq \sup _{z^{\prime}}\left\{u\left(x, z^{\prime}\right)-\varphi_{z}\left(z^{\prime}\right)\right\}-\varepsilon \\
& =\sup _{z^{\prime}}\left\{u\left(x, z^{\prime}\right)-u\left(x, z^{\prime}\right)+u(x, z)-p^{\sharp \sharp}(z)\right\}-\varepsilon \\
& =u(x, z)-p^{\sharp \sharp}(z)-\varepsilon=p^{\sharp}(x)-\varepsilon
\end{aligned}
$$

which is a contradiction. The result follows

Corollary 3. If $x$ is a point where $p^{\sharp}$ is differentiable, then:

$$
p\left(\nabla_{u} p^{\sharp}(x)\right)=p^{\sharp \sharp}\left(\nabla_{u} p^{\sharp}(x)\right)
$$

and:

$$
p^{\sharp}(x)=u\left(x, \nabla_{u} p^{\sharp}(x)\right)-p\left(\nabla_{u} p^{\sharp}(x)\right) .
$$

Proof. Just apply the preceding proposition, bearing in mind that $\partial p^{\sharp}(x)$ contains only one point, namely $\nabla_{u} p^{\sharp}(x)$. This yields equation (3.10). Equation (3.11) follows from the definition of the subgradient:

$$
p^{\sharp}(x)=u\left(x, \nabla_{u} p^{\sharp}(x)\right)-p^{\sharp}\left(\nabla_{u} p^{\sharp}(x)\right)
$$

and equation (3.10).

\section{6. $v$-concave functions}

Let us now consider the duality between $Y$ and $\dot{Z}$. Given $v: Y \times Z \rightarrow R$, we say that a map $g: Y \rightarrow \mathbb{R} \cup\{-\infty\}$ is $v$-concave iff there exists a non-empty subset $A \subset Z \times \mathbb{R}$ such that:

$$
\forall y \in Y, g(y)=\inf _{(z, \alpha) \in A}\{v(y, z)+a\}
$$

and a function $p: Z \rightarrow \mathbb{R} \cup\{-\infty\}$ will be called $v$-concave iff there exists a non-empty subset $B \subset X \times \mathbb{R}$ such that:

$$
p(z)=\inf _{(x, b) \in B}\{v(y, z)+b\}
$$


All the results on $u$-convex functions carry over to $v$-concave functions, with obvious modifications. The superconjugate of a function $g: Y \rightarrow \mathbb{R} \cup\{-\infty\}$, not identically $\{-\infty\}$, is defined by:

$$
g^{b}(z)=\inf _{y}\{v(y, z)-g(y)\}
$$

and the superconjugate of a function $p: Z \rightarrow \mathbb{R} \cup\{-\infty\}$, not identically $\{-\infty\}$, is given by:

$$
p^{b}(y)=\inf _{z}\{v(y, z)-p(z)\} .
$$

The superdifferential $\partial p^{b}$ is defined by:

$$
\partial p^{b}(y)=\arg \min _{z}\{v(y, z)-p(z)\}
$$

and we have the Fenchel inequality:

$$
p(z)+p^{b}(y) \leq v(y, z) \quad \forall(y, z)
$$

with equality iff $z \in \partial p^{b}(y)$. Note finally that $p^{b b} \geq p$, with equality if $p$ is $v$-concave

\section{The DuAl optimization PRoBlem}

Denote by $\mathcal{A}$ the set of all bounded function on $Z$ :

$$
p \in \mathcal{A} \Longleftrightarrow \sup _{z}|p(z)|<\infty
$$

and consider the minimization problem:

$$
\inf _{p \in \mathcal{A}}\left[\int_{X} p^{\sharp}(x) \mathrm{d} \mu-\int_{Y} p^{b}(y) \mathrm{d} \nu\right]
$$

Proposition 6. The minimum is attained in problem (4.1)

Proof. Take a minimizing sequence $p_{n}$ :

$$
\int_{X} p_{n}^{\sharp}(x) \mathrm{d} \mu-\int_{Y} p_{n}^{b}(y) \mathrm{d} \nu \rightarrow \inf (\mathrm{P}) .
$$

Setting $q_{n}=p_{n}+a$, for some constant $a$. Then $q_{n}^{\sharp}=p_{n}^{\sharp}-a$ and $q_{n}^{b}=p_{n}^{b}-a$. Since $\mu(X)=\nu(Y)$, we have:

$$
\int_{X} q_{n}^{\sharp}(x) \mathrm{d} \mu-\int_{Y} q_{n}^{b}(y) \mathrm{d} \nu=\int_{X} p_{n}^{\sharp}(x) \mathrm{d} \mu-\int_{Y} p_{n}^{b}(y) \mathrm{d} \nu \rightarrow \inf (\mathrm{P}) .
$$

Setting $a=-\inf _{z} p_{n}(z)$, we find $\inf _{z} q_{n}(z)=0$. So there is no loss of generality in assuming that:

$$
\forall z, \quad \inf _{z} p_{n}(z)=0
$$

which we shall do from now on.

The sequences $p_{n}^{\sharp}$ is $k$-Lipschitzian. Since $p_{n} \geq 0$, we have

$$
p_{n}^{\sharp}(x) \leq \sup _{z} u(x, z) \leq \max _{x, z} u(x, z) .
$$


Choose $z_{n}$ such that $p_{n}\left(z_{n}\right) \leq 1$. We then have:

$$
p_{n}^{\sharp}(x) \geq u\left(x, z_{n}\right)-p_{n}\left(z_{n}\right) \geq \min _{x, z} u(x, z)-1 .
$$

So the sequence $p_{n}^{\sharp}$ is uniformly bounded. By Ascoli's theorem, there is a uniformly convergent subsequence. Similarly, after extracting this first subsequence, we extract another one along which $p_{n}^{b}$ converges uniformly. The resulting subsequence will still be denoted by $p_{n}$, so that:

$$
\begin{aligned}
& p_{n}^{\sharp} \rightarrow f \text { uniformly } \\
& p_{n}^{b} \rightarrow g \text { uniformly. }
\end{aligned}
$$

Taking limits, we get:

$$
\int_{X} f(x) \mathrm{d} \mu-\int_{Y} g(y) \mathrm{d} \nu=\inf (\mathrm{P}) .
$$

It is easy to see that $p_{n}^{\sharp \sharp} \rightarrow f^{\sharp}$ and $p_{n}^{b b} \rightarrow g^{b}$ uniformly. Since $p_{n}^{\sharp \sharp} \leq p_{n}$ and $p_{n}^{b b} \geq p_{n}$, we have $p_{n}^{\sharp \sharp} \leq p_{n}^{b b}$, and hence $f^{\sharp} \leq g^{\text {b }}$. Set:

$$
\bar{p}=\frac{1}{2}\left(f^{\sharp}+g^{b}\right) .
$$

Since $f^{\sharp}$ and $g^{b}$ are Lipschitz continuous, so is $\bar{p}$. Since $f^{\sharp} \leq \bar{p} \leq g^{b}$, we must have $\bar{p}^{\sharp} \leq f^{\sharp} \leq f$ and $\bar{p}^{b} \geq g^{\text {bb }} \geq g$. Hence:

$$
\int_{X} \bar{p}^{\sharp}(x) \mathrm{d} \mu-\int_{Y} \bar{p}^{b}(y) \mathrm{d} \nu \leq \int_{X} f(x) \mathrm{d} \mu-\int_{Y} g(y) \mathrm{d} \nu=\inf (\mathrm{P}) .
$$

Since $f^{\sharp} \leq \bar{p} \leq g^{b}$, both sides being continuous functions, the function $\bar{p}$ must be bounded on $Z$, and the above inequality shows that it is a minimizer. The proof is concluded.

\section{Proof of the main Result}

Let us first express the optimality condition in problem $(\mathrm{P})$. Set:

$$
\begin{aligned}
& \bar{s}(x)=\nabla_{u} \bar{p}^{\sharp}(x) \\
& \bar{t}(y)=\nabla_{v} \bar{p}^{b}(y)
\end{aligned}
$$

where the gradient maps $\nabla_{u} \bar{p}^{\sharp}$ and $\nabla_{v} \bar{p}^{b}$ have been defined in proposition 4 .

Proposition 7. $\bar{s}(\mu)=\bar{t}(\nu)$.

Proof. We follow the argument in Carlier [2]. Take any continuous function $\varphi: Z \rightarrow R$. Since $\bar{p}$ is a minimizer, we have, for any integer $n$ :

$$
\int_{X} n\left[\left(\bar{p}+\frac{1}{n} \varphi\right)^{\sharp}-\bar{p}^{\sharp}\right] \mathrm{d} \mu-\int_{Y} n\left[\left(\bar{p}+\frac{1}{n} \varphi\right)^{b}-\bar{p}^{b}\right] \mathrm{d} \nu \geq 0 .
$$

We deal with the first integral. Set $\bar{p}+\frac{1}{n} \varphi=p_{n}$. Since $p_{n}^{\sharp}$ is $u$-convex, it is differentiable almost everywhere. Take a negligible subset $X_{0}$ such that all the $p_{n}^{\sharp}, n \in N$, and $\bar{p}^{\sharp}$, are differentiable at every $x \notin X_{0}$. If $x \notin X_{0}$, then $\nabla_{u} p_{n}^{\sharp}(x)$ is the only point in $\partial p_{n}^{\sharp}(x)$, and we have, by Corollary 3 :

$$
u\left(x, \nabla_{u} p_{n}^{\sharp}(x)\right)-\bar{p}\left(\nabla_{u} p_{n}^{\sharp}(x)\right) \leq \bar{p}^{\sharp}(x)=u\left(x, \nabla_{u} \bar{p}^{\sharp}(x)\right)-\bar{p}\left(\nabla_{u} \bar{p}^{\sharp}(x)\right)
$$

so that:

$$
u\left(x, \nabla_{u} p_{n}^{\sharp}(x)\right)-\bar{p}\left(\nabla_{u} p_{n}^{\sharp}(x)\right)-u\left(x, \nabla_{u} \bar{p}^{\sharp}(x)\right)+\bar{p}\left(\nabla_{u} \bar{p}^{\sharp}(x)\right) \leq 0
$$


yielding:

$$
p_{n}^{\sharp}(x)+\frac{1}{n} \varphi\left(\nabla_{u} p_{n}^{\sharp}(x)\right)-\bar{p}^{\sharp}(x) \leq 0 .
$$

From the definition of $p_{n}^{\sharp}$, we have, using Corollary 3 again:

$$
u\left(x, \nabla_{u} \bar{p}^{\sharp}(x)\right)-\bar{p}\left(\nabla_{u} \bar{p}^{\sharp}(x)\right)-\frac{1}{n} \varphi\left(\nabla_{u} \bar{p}^{\sharp}(x)\right) \leq p_{n}^{\sharp}(x)=u\left(x, \nabla_{u} p_{n}^{\sharp}(x)\right)-\bar{p}\left(\nabla_{u} p_{n}^{\sharp}(x)\right)-\frac{1}{n} \varphi\left(\nabla_{u} p_{n}^{\sharp}(x)\right) .
$$

Rewriting this, we get:

$$
\begin{aligned}
\frac{1}{n} \varphi\left(\nabla_{u} p_{n}^{\sharp}(x)\right)-\frac{1}{n} \varphi\left(\nabla_{u} \bar{p}^{\sharp}(x)\right) & \leq u\left(x, \nabla_{u} p_{n}^{\sharp}(x)\right)-\bar{p}\left(\nabla_{u} p_{n}^{\sharp}(x)\right)-u\left(x, \nabla_{u} \bar{p}^{\sharp}(x)\right)+\bar{p}\left(\nabla_{u} \bar{p}^{\sharp}(x)\right) \\
& =p_{n}^{\sharp}(x)+\frac{1}{n} \varphi\left(\nabla_{u} p_{n}^{\sharp}(x)\right)-\bar{p}^{\sharp}(x)
\end{aligned}
$$

yielding:

$$
-\frac{1}{n} \varphi\left(\nabla_{u} \bar{p}^{\sharp}(x)\right) \leq p_{n}^{\sharp}(x)-\bar{p}^{\sharp}(x) .
$$

Inequalities (5.3) and (5.6) together give:

$$
-\varphi\left(\nabla_{u} \bar{p}^{\sharp}(x)\right) \leq n\left(p_{n}^{\sharp}(x)-\bar{p}^{\sharp}(x)\right) \leq-\varphi\left(\nabla_{u} p_{n}^{\sharp}(x)\right) .
$$

Now let $n \rightarrow \infty$. Using corollary 3 , we have:

$$
u\left(x, \nabla_{u} p_{n}^{\sharp}(x)\right)=p_{n}\left(\nabla_{u} p_{n}^{\sharp}(x)\right)+p_{n}^{\sharp}(x) .
$$

Since $Z$ is compact, the sequence $\nabla_{u} p_{n}^{\sharp}(x) \in Z$ has a cluster point $z$, and since $p_{n}$ and $p_{n}^{\sharp}$ converge to $\bar{p}$ and $\bar{p}^{\sharp}$ uniformly, we get in the limit:

$$
u(x, z)=\bar{p}(z)+\bar{p}^{\sharp}(x)
$$

so that $z \in \partial \bar{p}^{\sharp}(x)$. But that subdifferential consists only of the point $\nabla_{u} \bar{p}^{\sharp}(x)$, so that $z=\nabla_{u} \bar{p}^{\sharp}(x)$. This shows that the cluster point $z$ is unique, so that the whole sequence must converge:

$$
\nabla_{u} p_{n}^{\sharp}(x) \rightarrow \nabla_{u} \bar{p}^{\sharp}(x) .
$$

Letting $n \rightarrow \infty$ in (5.7), we get:

$$
\forall x \notin X_{0}, \lim _{n} n\left(p_{n}^{\sharp}(x)-\bar{p}^{\sharp}(x)\right)=-\varphi\left(\nabla_{u} \bar{p}^{\sharp}(x)\right) .
$$

Similarly, we have:

where $Y_{0} \subset Y$ is negligible.

$$
\forall y \notin Y_{0}, \lim _{n} n\left(p_{n}^{b}(x)-\bar{p}^{b}(x)\right)=-\varphi\left(\nabla_{u} \bar{p}^{b}(x)\right)
$$

Since $\varphi$ is continuous, it is bounded, and we can apply the dominated convergence theorem to inequality (5.1). We get:

$$
-\int_{X} \varphi\left(\nabla_{u} \bar{p}^{\sharp}(x)\right) \mathrm{d} \mu+\int_{Y} \varphi\left(\nabla_{u} \bar{p}^{b}(y)\right) \mathrm{d} \nu \geq 0 .
$$

Since the inequality must hold for $-\varphi$ as well as $\varphi$, it is in fact an equality. In other words, for any $\varphi: Z \rightarrow R$ with compact support, we have:

$$
\int_{X} \varphi\left(\nabla_{u} \bar{p}^{\sharp}(x)\right) \mathrm{d} \mu=\int_{Y} \varphi\left(\nabla_{u} \bar{p}^{b}(y)\right) \mathrm{d} \nu
$$

and this means that $\bar{s}(\mu)=\bar{t}(\nu)$, as announced. 
Set $\bar{s}(\mu)=\bar{t}(\nu)=\lambda$. This is a positive measure on $Z$, not necessarily absolutely continuous with respect to the Lebesgue measure.

Applying Corollary 3, we have:

$$
\begin{aligned}
& \bar{p}^{\sharp}(x)=u(x, \bar{s}(x))-\bar{p}(\bar{s}(x)) \\
& \bar{p}^{b}(y)=v(y, \bar{t}(y))-\bar{p}(\bar{t}(y))
\end{aligned}
$$

and hence:

$$
\begin{aligned}
\int_{X} \bar{p}^{\sharp}(x) \mathrm{d} \mu-\int_{Y} \bar{p}^{b}(y) \mathrm{d} \nu & =\int_{X}[u(x, \bar{s}(x))-\bar{p}(\bar{s}(x))] \mathrm{d} \mu-\int_{Y}[v(y, \bar{t}(y))-\bar{p}(\bar{t}(y))] \\
& =\int_{X} u(x, \bar{s}(x)) \mathrm{d} \mu-\int_{Y} v(y, \bar{t}(y)) \mathrm{d} \nu-\left[\int_{X} \bar{p}(\bar{s}(x)) \mathrm{d} \mu-\int_{Y} \bar{p}(\bar{t}(y)) \mathrm{d} \nu\right] \\
& =\int_{X} u(x, \bar{s}(x)) \mathrm{d} \mu-\int_{Y} v(y, \bar{t}(y)) \mathrm{d} \nu-\left[\int_{Z} \bar{p}(z) \mathrm{d} \lambda-\int_{Z} \bar{p}(z) \mathrm{d} \lambda\right] \\
& =\int_{X} u(x, \bar{s}(x)) \mathrm{d} \mu-\int_{Y} v(y, \bar{t}(y)) \mathrm{d} \nu .
\end{aligned}
$$

Let $(s, t)$ be a pair of Borelian maps such that $s(\mu)=t(\nu)$. Then, by the Fenchel inequality:

$$
\begin{aligned}
\int_{X} u(x, s(x)) \mathrm{d} \mu-\int_{Y} v(y, t(y)) \mathrm{d} \nu & \leq \int_{X}\left[\bar{p}^{\sharp}(x)+\bar{p}(s(x))\right] \mathrm{d} \mu-\int_{Y}\left[p^{b}(y)+\bar{p}(t(y))\right] \mathrm{d} \nu \\
& =\int_{X} \bar{p}^{\sharp}(x) \mathrm{d} \mu-\int_{Y} \bar{p}^{b}(y) \mathrm{d} \nu+\left[\int_{X} \bar{p}(s(x)) \mathrm{d} \mu-\int_{Y} \bar{p}(t(y)) \mathrm{d} \nu\right] .
\end{aligned}
$$

The last bracket vanishes because $s(\mu)=t(\nu)$. Applying inequality 5.9, we get:

$$
\int_{X} u(x, s(x)) \mathrm{d} \mu-\int_{Y} v(y, t(y)) \mathrm{d} \nu \leq \int_{X} u(x, \bar{s}(x)) \mathrm{d} \mu-\int_{Y} v(y, \bar{t}(y)) \mathrm{d} \nu .
$$

This shows that $(\bar{s}, \bar{t})$ is a maximizer, and proves the existence part of Theorem 1 .

As for uniqueness, assume that there is another maximizer $\left(s^{\prime}, t^{\prime}\right)$. The preceding inequality then becomes an equality:

$$
\int_{X} u(x, s(x)) \mathrm{d} \mu-\int_{Y} v(y, t(y)) \mathrm{d} \nu=\int_{X}\left[\bar{p}^{\sharp}(x)+\bar{p}(s(x))\right] \mathrm{d} \mu-\int_{Y}\left[p^{b}(y)+\bar{p}(t(y))\right] \mathrm{d} \nu
$$

which we rewrite as:

$$
\int_{X}\left[\bar{p}^{\sharp}(x)+\bar{p}(s(x))-u(x, s(x))\right] \mathrm{d} \mu+\int_{Y}\left[v(y, t(y))-p^{b}(y)-\bar{p}(t(y))\right] \mathrm{d} \nu=0 .
$$

Both integrands are non-negative by the Fenchel inequality. If the sum is zero, each integral must vanish, and since the integrands are non-negative, each integrand must vanish almost everywhere. This means that:

$$
\begin{aligned}
& s(x) \in \partial \bar{p}^{\sharp}(x) \text { a.e. } \\
& t(y) \in \partial \bar{p}^{b}(y) \quad \text { a.e. }
\end{aligned}
$$

and since $\partial \bar{p}^{\sharp}(x)=\{\bar{s}(x)\}$ and $\partial \bar{p}^{b}(y)=\{\bar{t}(y)\}$ almost everywhere, the uniqueness part of Theorem 1 is proved. 


\section{A DEgenerate CASE}

We shall now investigate some properties of the function $\bar{p}: Z \rightarrow R$.

Recall that we denote $\lambda=\bar{s}(\mu)=\bar{t}(\nu)$. It is a positive measure on $Z$. Its support $\operatorname{Supp}(\lambda)$ is the complement of the largest open subset $\Omega \subset Z$ such that $\lambda=0$ on $\Omega$. If for instance $\mu$ and $\nu$ have the property that the measure of any open non-empty subset is positive, then:

$$
\operatorname{Supp}(\lambda)=\bar{s}(X)=\bar{t}(Y) \text {. }
$$

Proposition 8. $\bar{p}^{\sharp \sharp}=\bar{p}=\bar{p}^{\text {bb }}$ on $\operatorname{Supp}(\lambda)$.

Proof. We have seen that $\bar{p}^{\sharp \sharp}(\bar{s}(x))=\bar{p}(\bar{s}(x)) \mu$-almost everywhere. Since $\bar{s}(\mu)=\lambda$, this means that $\bar{p}^{\sharp \sharp}(z)=$ $\bar{p}(z) \lambda$-almost everywhere. Since $\bar{p}$ and $\bar{p}^{\sharp \sharp}$ are continuous, equality extends to the support of $\lambda$. Similarly, $\bar{p}=\bar{p}^{\text {bb }}$ on $\operatorname{Supp}(\lambda)$, and the result follows.

In certain cases, proposition 8 will impose strong restrictions on $p$. Let us give an example.

Example 3. Suppose $X, Y, Z$ are compact subsets of $R^{n}$, and we want to minimize:

$$
\int_{X}\|x-s(x)\|^{2} \mathrm{~d} \mu-\int_{Y}\|y-t(y)\|^{2} \mathrm{~d} \nu
$$

among all maps $(s, t)$ such that $s(\mu)=t(\nu)$.

Developing the squares, this amounts to minimizing:

$$
\left[\int_{X} x^{2} \mathrm{~d} \mu-\int_{Y} y^{2} \mathrm{~d} \nu\right]+\left[\int_{X} s(x)^{2} \mathrm{~d} \mu-\int_{Y} t(y)^{2} \mathrm{~d} \nu\right]-\left[\int_{X} x^{\prime} s(x) \mathrm{d} \mu-\int_{Y} y^{\prime} t(y) \mathrm{d} \nu\right] .
$$

The first bracket is a constant (it does not depend on the choice of $s$ and $t$ ). The second bracket vanishes because $s(\mu)=t(\nu)$. We are left with the last one. So the problem amounts to maximizing:

$$
\int_{X} x^{\prime} s(x) \mathrm{d} \mu-\int_{Y} y^{\prime} t(y) \mathrm{d} \nu
$$

and it falls within the scope of Theorem 1 by setting $u(x, z)=x^{\prime} z$ and $v(y, z)=y^{\prime} z$. Then $u$-convex functions are convex in the usual sense, $v$-concave functions are concave in the usual sense. By proposition $8, p$ is linear on $\operatorname{Supp}(\lambda)$, so we may take:

$$
p(z)=\pi^{\prime} z
$$

for some vector $\pi \in R^{n}$. We then get $\bar{s}(x)$ by maximizing $(x-\pi)^{\prime} z$ over $Z$. Similarly, we get $\bar{t}(y)$ by minimizing $(y-\pi)^{\prime} z$ over $Z$. Note that this implies that $\bar{s}(X)=\bar{t}(Y) \subset \partial Z$, the boundary of $Z$. Let us summarize:

Proposition 9. There is a single map $(\bar{s}, \bar{t})$ which minimizes the integral (6.1) among all maps $(s, t)$ such that $s(\mu)=t(\nu)$. It is given by:

$$
\begin{aligned}
& \bar{s}(x)=\arg \max _{z}(x-\pi)^{\prime} z \\
& \bar{t}(y)=\arg \min _{z}(y-\pi)^{\prime} z
\end{aligned}
$$

and the actual value of $\pi \in R^{n}$ is found by substituting (6.2) and (6.3) in the integral (6.1) and by minimizing the resulting function of $\pi$.

It follows that the image $\bar{s}(X)=\bar{t}(Y)=\operatorname{Supp}(\lambda)$ is contained in the boundary of $Z$. This is an example of degeneracy: the dimension of $\operatorname{Supp}(\lambda)$ is strictly less than the dimension $n$ of $X, Y$ and $Z$, so that the maps $\bar{s}$ and $\bar{t}$ cannot be one-to-one. 


\section{THE NON-DEGENERATE CASE}

Suppose $X$ and $Y$ are subsets of $R^{n}$ endowed with the Lebesgue measure, and that there is a subset $Z_{0} \subset$ $Z$ such that $\bar{s}(X)=\bar{t}(Y)=Z_{0}=\operatorname{Supp}(\lambda)$, and suppose moreover that there are $C^{1}$ maps $\sigma: Z_{0} \rightarrow X$ and $\tau: Z_{0} \rightarrow Y$ such that:

$$
\bar{s} \circ \sigma=I_{Z_{0}}=\bar{t} \circ \tau .
$$

The maps $\sigma, \tau$ and the subset $Z_{0}$ are related by:

$$
\begin{aligned}
\tau \circ \sigma^{-1}(\mu) & =\nu \\
\sigma\left(Z_{0}\right) & =X \\
\tau\left(Z_{0}\right) & =Y .
\end{aligned}
$$

On the other hand, $\sigma(z)$ is the subgradient of $\bar{p}$ at $z$, and $\tau(z)$ is the supergradient of $\bar{p}$ at $z$, so that:

$$
\begin{aligned}
\bar{p}^{\sharp}(\sigma(z)) & =u(\sigma(z), z)-\bar{p}(z) \\
\bar{p}^{b}(\tau(z)) & =v(\tau(z), z)-\bar{p}(z)
\end{aligned}
$$

for all $z \in Z_{0}$. By proposition $8, p=\bar{p}^{\sharp}=p^{b}$, so that $\bar{p}$ is $C^{1}$ on $Z_{0}$. The derivative $D_{z} \bar{p}$ is related to $\sigma$ and $\tau$ by the equations

$$
\begin{aligned}
& D_{z} u(\sigma(z), z)=D_{z} \bar{p}(z) \\
& D_{z} v(\tau(z), z)=D_{z} \bar{p}(z) .
\end{aligned}
$$

Inverting these relations, and replacing $\sigma$ and $\tau$ by their expression in terms of $D_{z} \bar{p}$ in the relations (7.1)-(7.3), one gets a series of conditions on $D_{z} \bar{p}$. The first one turns out to be a second-order equation on Monge-Ampere type, while the two others are boundary conditions.

Example 4. Consider the problem of minimizing the integral:

$$
\int_{X} \frac{\alpha}{2}\|x-s(x)\|^{2} \mathrm{~d} x+\int_{Y} \frac{1}{2}\|y-t(y)\|^{2} \mathrm{~d} y
$$

over all maps $(s, t)$ such that $s(\mu)=t(\nu)$. Here $\alpha>0$ is a given constant.

We apply Theorem 1 with $u(x, z)=-\frac{\alpha}{2}\|x-z\|^{2}$ and $v(y, z)=\frac{1}{2}\|y-z\|^{2}$. Note that the function $p: Z \rightarrow R$ is $u$-convex iff $D_{z z}^{2} p(z) \geq-\alpha I$ for every $z$, where $D_{z z}^{2} p(z)$ is the Hessian matrix at $z$, and that it is $v$-concave iff $D_{z z}^{2} p(z) \leq I$. So there a function $p: Z \rightarrow R$ is both $u$-convex and $v$-concave if and only if it satisfies $-\alpha I \leq D_{z z}^{2} p(z) \leq I$ everywhere. There are many such functions, so the condition $p^{\sharp \sharp}=p=p^{\text {bb }}$ in Proposition 8 is no longer binding.

Equations (7.4) and (7.5) become:

$$
\begin{aligned}
& D_{z} \bar{p}(z)=-\alpha(\sigma(z)-z) \\
& D_{z} \bar{p}(z)=(\tau(z)-z) .
\end{aligned}
$$


So $\sigma(z)=z-\frac{1}{\alpha} D_{z} \bar{p}(z)$ and $\tau(z)=z+D_{z} \bar{p}(z)$. The system (7.1)-(7.3) becomes:

$$
\begin{aligned}
& \operatorname{det}\left[I+D_{z z}^{2} \bar{p}(z)\right]= \pm \operatorname{det}\left[I-\frac{1}{\alpha} D_{z z}^{2} \bar{p}(z)\right] \\
& \forall z \in Z_{0}, \quad z-\frac{1}{\alpha} D_{z} \bar{p}(z) \in X \\
& \forall z \in Z_{0}, \quad z+D_{z} \bar{p}(z) \in Y .
\end{aligned}
$$

Note that, since the measure of $\sigma(X)$ equals the measure of $X$, condition (7.8), which states that $\sigma(X) \subset X$, implies that in fact $\sigma(X)=X$. Similarly, condition (7.9) implies that $\tau(X)=X$.

Example 5. Given $a>2$, consider the problem of minimizing the integral:

$$
\int_{1}^{2}\left[\frac{1}{2} s(x)^{2}-x s(x)\right] \mathrm{d} x+\int_{a}^{a+1} \frac{1}{2} y t(y)^{2} \mathrm{~d} y
$$

over all maps $s:[1,2] \rightarrow[0,1]$ and $t:[a, a+1] \rightarrow[0,1]$ such that $s(\rho)=t(\rho)$, where $\rho$ is the Lebesgue measure.

This is the framework of Theorem 1 with $X=[1,2], Y=[a, a+1], Z=[0,1]$ and $u(x, z)=-\frac{1}{2} z^{2}+x z$, $v(y, z)=\frac{1}{2} y z^{2}$.

However, it is a case when the direct approach mentioned in the introduction will work. Define a function $f: X \times Y \rightarrow R$ by:

$$
\begin{aligned}
f(x, y) & =\max _{0 \leq z \leq 1}\left\{-\frac{1}{2} z^{2}+x z-\frac{1}{2} y z^{2}\right\} \\
& =\left\{-\frac{1}{2} z^{2}+x z-\frac{1}{2} y z^{2} \mid z=\frac{x}{1+y}\right\} \\
& =\frac{1}{2} \frac{x^{2}}{1+y} .
\end{aligned}
$$

We seek to maximize the integral:

$$
I(\varphi)=\int_{1}^{2} f(x, \varphi(x)) \mathrm{d} x
$$

among all measure-preserving maps $\varphi:[1,2] \rightarrow[a, a+1]$. Assuming that the solution is continuous, we find two candidates, $\varphi_{1}$ and $\varphi_{2}$, given by:

$$
\begin{aligned}
& \varphi_{1}(x)=x+a-1 \\
& \varphi_{2}(x)=-x+a+2
\end{aligned}
$$

and a direct calculation yields $I\left(\varphi_{2}\right)>I\left(\varphi_{1}\right)$. The solution is given by

$$
\begin{aligned}
& s_{2}(x)=\frac{x}{1+\varphi_{2}(x)}=\frac{x}{3+a-x} \\
& t_{2}(y)=\frac{\varphi_{2}^{-1}(y)}{1+y}=\frac{a+2-y}{1+y}
\end{aligned}
$$


so that $Z_{0}=\left[\frac{1}{a+2}, \frac{2}{a+1}\right]$. These equations can be inverted, yielding:

$$
\begin{aligned}
& x=\frac{z}{1+z}(a+3) \\
& y=\frac{a+2-z}{1+z} .
\end{aligned}
$$

The potential $p(z)$ is found by maximizing $u(x, z)-p(z)$ or minimizing $v(y, z)-p(z)$ with respect to $z$, yielding

$$
\begin{aligned}
& x=\sigma(z)=p^{\prime}(z)+z \\
& y=\tau(z)=p^{\prime}(z) / z
\end{aligned}
$$

into which we substitute equations (7.10) and (7.11), finding:

$$
p^{\prime}(z)=\frac{z}{1+z}(a+2-z)
$$

Acknowledgements. The author thanks Jim Heckman for introducing him to the economics of hedonic pricing.

\section{REFERENCES}

[1] Y. Brenier, Polar factorization and monotone rearrangements of vector-valued functions. Comm. Pure App. Math. 44 (1991) $375-417$.

[2] G. Carlier, Duality and existence for a class of mass transportation problems and economic applications, Adv. Math. Economics 5 (2003) 1-21.

[3] I. Ekeland and R. Temam, Convex analysis and variational problems. North-Holland Elsevier (1974) new edition, SIAM Classics in Appl. Math. (1999).

[4] W. Gangbo and R. McCann, The geometry of optimal transportation. Acta Math. 177 (1996) 113-161.

[5] I. Ekeland, J. Heckman and L. Nesheim, Identification and estimation of hedonic models. J. Political Economy 112 (2004) 60-109.

[6] L. Kantorovitch, On the transfer of masses, Dokl. Ak. Nauk USSR 37 (1942) 7-8.

[7] S. Rachev and A. Ruschendorf, Mass transportation problems. Springer-Verlag (1998).

[8] C. Villani, Topics in mass transportation. Grad. Stud. Math. 58 (2003) 